\title{
Correlation of Lamina Cribrosa and Standard Automated Perimetry Findings in Glaucoma and Non- Glaucoma Patients
}

\author{
Ian J Daguman* and Manuel S Delfin \\ Department of Ophthalmology, Manila Doctors Hospital, UN Ave, Manila, Philippines
}

${ }^{*}$ Corresponding author: Daguman IJ, Department of Ophthalmology, Manila Doctors Hospital, UN Ave., Manila 1000, Philippines, Tel: +639175735354, +639985311787; E-mail: ian.daguman@gmail.com

Received: 15 Mar, 2018 | Accepted: 07 Jun, 2018 | Published: 14 Jun, 2018

Citation: Daguman IJ, Delfin MS (2018) Correlation of Lamina Cribrosa and Standard Automated Perimetry Findings in Glaucoma and NonGlaucoma Patients. J Ophthalmic Stud 2(1): dx.doi.org/10.16966/2639-152X.110

Copyright: () 2018 Daguman IJ, et al. This is an open-access article distributed under the terms of the Creative Commons Attribution License, which permits unrestricted use, distribution, and reproduction in any medium, provided the original author and source are credited.

\begin{abstract}
Purpose: To assess the correlation between lamina cribrosa thickness and standard automated perimetry results in healthy and glaucomatous eyes.

Methods: This is a single center, single observer, cross-sectional study. Eyes were categorized as glaucomatous or non-glaucomatous using the Lamina Cribrosa Exploration Study (Bundang Hospital Glaucoma Clinic of the Seoul National University). The glaucoma group was further subcategorized by their severity (mild, moderate or severe) based on the modified Hodapp-Anderson-Parish criteria. Visual field testing was done using Humphrey Field Analyzer Cirrus 5000 (Swedish Interactive Threshold Algorithm 30-2; Carl Zeiss Meditec, Dublin, CA). The Institutional Review Board of the Manila Doctors Hospital approved this study.
\end{abstract}

Main Outcome Measures: The lamina cribrosa thickness and glaucoma severity. Correlations between the lamina cribrosa thickness and visual field test results of glaucomatous and non-glaucomatous eyes were analyzed using Spearman correlation. Comparisons of lamina cribrosa thickness between glaucomatous and non-glaucomatous eyes were analyzed with independent t-test. Comparisons of lamina cribrosa thickness across glaucoma severities were analyzed using analysis of variance (ANOVA). $5 \%$ level of significance was employed.

Results: Forty five (45) eyes were included in the study. There was a statistically significant difference in the lamina cribrosa thickness between nonglaucomatous and moderate glaucoma groups $(t=1.89, p=0.034)$. There were no significant differences in the lamina cribrosa thickness between non-glaucomatous and mild glaucoma groups ( $t=1.198, p=0.1198)$, as well as between mild and moderate glaucoma groups $(t=0.726, p=0.2389)$. There is no strong correlation between the lamina cribrosa depth and lamina cribrosa thickness to the severity of visual field test results (LC depth $r=0.239, p=0.102)$, ( $L C$ thickness $r=0.160, p=0.274)$. Furthermore, it was found that there is no significant difference in the lamina cribrosa thickness across the different glaucoma severities ( $f$-ratio $=3.408, p=0.0556)$.

Conclusion: Lamina cribrosa thickness was significantly different between non- glaucomatous and moderate glaucoma eyes. It may be used as another index in diagnosing moderate glaucomatous damage or monitoring glaucoma progression.

Keywords: Lamina cribrosa depth; Visual field severity; Laminar thickness; OCT; Glaucoma

\section{Introduction}

Glaucoma is the leading cause of irreversible blindness worldwide [1]. According to the World Health Organization (WHO), it ranks as the second leading cause of visual impairment globally [2]. The magnitude of glaucoma as a disease is evident in the quality of life of glaucoma patients who suffer greatly due to its irreversible progression. Locally, it is the third leading cause of bilateral blindness [3]. Glaucoma is a visual field (VF) defect which is accompanied by a characteristic optic neuropathy [4]. Early diagnosis and classification of glaucoma severity is vital in tailoring treatment that is appropriate for each patient to delay irreversible visual impairment. Currently, the standard in the management for patients with glaucoma is not solely based on clinical findings. Gamuts of ancillary tests are done to diagnose, monitor progression and manage response to treatment of glaucoma patients. Glaucoma is a progressive optic neuropathy with chronic neurodegenerative degeneration of the axons in the retinal ganglion cell (RGC) layer [5], leading to visual field loss. However, there is a subset of glaucoma patients that does not manifest with VF defects, these patients would only show ocular structural changes especially in the optic nerve head (ONH) [6]. In the past two decades, ophthalmologists have not only looked into the optic nerve structure, but have also developed imaging modalities that assess the function of the eye and correlate it with different eye structures.

\section{Review of Related Literature}

There is a growing body of knowledge that points to glaucomatous impairment of the visual system from damage of RGC axons within 
the lamina cribrosa area. Exploring the lamina cribrosa with the use of enhanced depth imaging-optical coherence tomography (EDI-OCT) technique has been recently employed. Initially, this technology is used to assess the choroid in the macular area, now there has been an expanding interest looking into lamina cribrosa in-vivo mainly because it responds dynamically to intraocular pressure (IOP) changes [5]. This study is limited with inferior resolution and contrast of OCT images compared to histology or second harmonic generated imaging [6]. Furthermore, OCT has not been proven capable in imaging the whole lamina cribrosa with good reproducibility. Delineation of the posterior surface of the lamina is highly variable even with EDI technology and expert's opinion. A considerable region of the lamina cribrosa remains difficult to visualize. The detection of the posterior surface of the lamina cribrosa in OCT images must still be verified by comparison with histology, [7] which is the gold standard. However, it is necessary to understand how OCT defined measurements of laminar thickness and relate it to visual function in glaucoma.

Current OCT technology and software reaches deeper structures. There have been studies that related the lamina cribrosa thickness to be thinner in glaucoma patients than in normal subjects [8]. It has been shown that the collagen in the lamina cribrosa increases throughout life and the laminar beam thickness increases with increasing age [9-12]. Further study including large numbers of normal subjects is needed. Glaucoma diagnosis and prognostication has utilized clinical, structural and functional findings of the patients' eyes. Indices have been formulated and currently being analyzed to support theories that certain structures, such as the retinal nerve fiber layer (RNFL) and ganglion cell layer (GCL) thickness, can be an indicator to help clinicians properly address glaucoma management.

\section{General objective}

- To assess the correlation of lamina cribrosa thickness and standard automated perimetry (SAP) findings in glaucoma and non-glaucoma patients in a tertiary hospital.

\section{Specific objectives}

- To compare laminar tissue thickness between glaucoma and non-glaucoma patients.

- To compare the difference of the pre-laminar and the laminar tissue thickness between the different glaucoma categories.

- To compare pre-laminar and laminar tissue thickness of glaucomatous and non-glaucomatous eyes.

\section{Study Design}

- This study is a single center, single observer, cross-sectional study.

\section{Sampling Technique}

Subjects will be gathered through period prevalence sampling, basing on the records of the patients who have undergone OCT and SAP not 6 months apart in the vision center of a tertiary hospital. The sampling criteria based on the Lamina Cribrosa Exploration Study of the Bundang Hospital Glaucoma Clinic of the Seoul National University will be adapted to select appropriate subjects to be included in the study. Patients' records will be reviewed by a single observer.

Glaucoma subjects are defined as patients with glaucomatous optic nerve damage with visual field defect without other ocular disease or conditions that cause increase in IOP. Non- glaucoma subjects are patients with IOP of less than $22 \mathrm{mmHg}$, normal visual fields and normal optic discs.

\section{Inclusion criteria}

- Open anterior chamber angle,

- Best corrected visual acuity (BCVA) of $20 / 40$ or better,

- Refraction of +/- 6 diopters spherical equivalent, and/or with +/3 diopters of cylinder,

- Clear anterior chamber, clear ocular media, no significant cataract of up to nuclear opacity 3, cortical grade of 3, and posterior capsular opacity of 2 based on Lens Opacity Classification System III (LOCS III) criteria,

- SAP results with reliable results (false $+/$ - of less than $15 \%$, fixation loss of less than 20\%),

- OCT of ONH with good signal strength of at least 7/10.

\section{Exclusion criteria}

- History of laser surgery or intraocular surgery except for cataract surgery,

- Secondary causes of elevation in IOP,

- Co-existing retinal or neurologic disorders.

\section{Methodology}

Once a subject undergoes SAP and OCT of ONH, patient will be included in the study, patient records will be reviewed and criteria will be applied. If patient is eligible, patient's data will then be included for the study.

Subjects will then be grouped into two main groups namely glaucoma and non-glaucoma groups. The glaucoma group will be composed of patients diagnosed by three glaucoma specialists $(\mathrm{MD}, \mathrm{JV}, \mathrm{AB})$. These are the subjects who have glaucomatous optic nerve damage with visual field defects without secondary causes of increase IOP. Glaucomatous optic nerve damage is defined with vertical cup-to-disc (CDR) of at least 0.7 , asymmetry of vertical CDR by 0.2 or more between eyes, focal neuroretinal thinning, notching of vessels, disc hemorrhage. Glaucomatous visual field change is defined as having a Humphrey SAP reading of global hemifield test (GHT) of outside normal limit, pattern deviation of at least 3 non-edged points with below the $5 \%$ and at least 1 point below $1 \%$, pattern standard deviation (PSD) of at least $5 \%$. The non-glaucoma group will be composed of patients with healthy eyes, glaucoma suspects whose IOP are less than $22 \mathrm{mmHg}$, normal visual fields and normal optic discs, with no visually impairing retinal or neurologic disorders. Main outcome measures are lamina cribrosa thickness and SAP findings. Lamina cribrosa thickness will be measured from the OCT B-scan images of the $\mathrm{ONH}$, specifically using 5-line raster of the Cirrus HD-OCT 5000 (Carl Zeiss Meditec, Dublin, CA, USA) with EDI and FastTrac modes on. These images will be measured using the image analyzer software which built-in the Zeiss Forum, its manual caliper will be used to determine the pre- laminar and laminar depths, wherein the lamina cribrosa thickness, and prelaminar thickness can be computed. Based on the high definition images of the ONH 5-line raster, the deepest part of the optic nerve pit will be used. A reference line is determined by connecting the two terminations of the RPE-Bruch's membrane (Figure 1). Prelaminar area is the homogenous area at the surface of the optic nerve pit. Underneath it is the lamina cribrosa, located by identifying the hyper reflective structure with hypo reflective columns, representing the laminar pores (Figure 2), the end of the hyper reflective signal is the posterior border of the lamina cribrosa (Figure 3). 

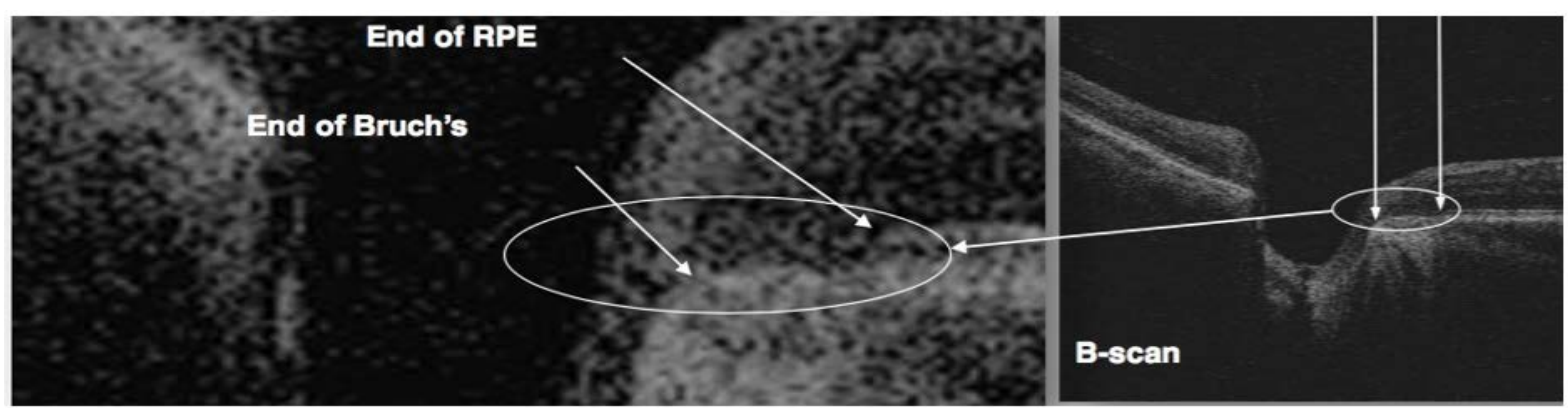

Figure 1: B-scan image of the terminations of RPE and Bruch's membran (used as reference point).

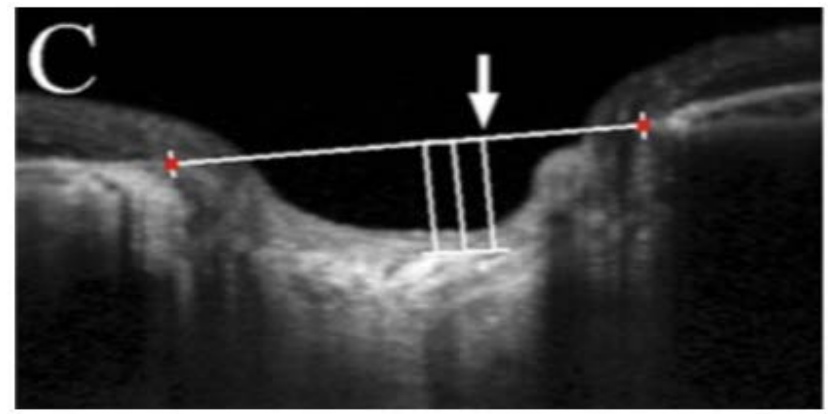

Figure 2: Anterior lamina cribrosa border.

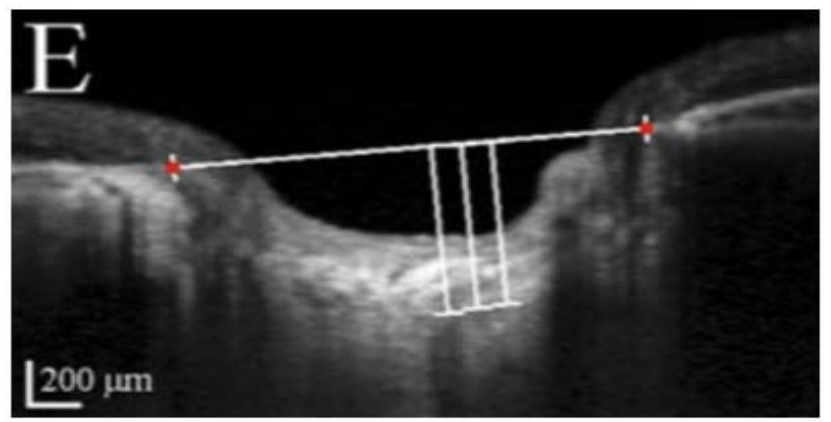

Figure 3: Posterior lamina cribrosa border.

SAP findings will be gathered on reliable automated visual field tests of the patient. The Humphrey visual field analyzer (Carl Zeiss Meditec, Dublin, CA, USA), full threshold, 30-2 SITA strategy will be used. The glaucoma group will be further categorized into different glaucoma severities (mild, moderate or severe) using modified Hodapp-Anderson- Parish criteria (Table 1). The Institutional Review Board (IRB) of the Manila Doctors Hospital approved this study, and the study design followed the principles of the Declaration of Helsinki. The study will look into the results of patients OCT of the ONH and SAP tests which are done in a non- invasive, non-contact testing. There are no foreseeable risks to the subjects during the study. Data will be gathered from diagnostic tests and medical records of patients. Follow-ups will not be necessary. There is no direct benefit to any participant. Establishment of the lamina cribrosa thickness is a recent index in assessing glaucoma progression and detection has not been widely accepted as more studies are still ongoing. Study subjects will not receive any incentive, in cash or kind.

\section{Statistical Analysis}

For the sample size computation, software Stata SE Version [13] was used to calculate and it showed a minimum requirement of gathering 18 subjects. Correlation between the lamina cribrosa thickness and severity of glaucoma will be analyzed using Spearman correlation. Correlation of laminar and pre-laminar thickness among glaucoma patients will be analyzed using dependent t-test. Comparison of lamina cribrosa thickness between glaucoma and non-glaucoma groups will be analyzed with independent t-test. Comparison of laminar thickness across glaucoma severities will be analyzed using analysis of variance (ANOVA). 5\% level of significance will be employed.

\section{Results}

The data collected came from 48 eyes of 28 patients. Baseline characteristics of the subjects are presented in (Table 2). Glaucoma subjects were further categorized into different severities as see in (Table 3). There was a significant difference in the lamina cribrosa thickness between glaucomatous and non-glaucomatous eyes ( $t=-$ $1.842, p=0.036)$. However, there is no significant difference in the prelaminar thickness between glaucomatous and non-glaucomatous eyes ( $t=-0.704, p=0.2425)$. There is also no significant difference between the lamina cribrosa thickness across the different glaucoma severities $(f$-ratio $=3.408, p=0.0556)$. There is no strong correlation between the lamina cribrosa depth and thickness to the severity of visual field test results (LC depth $r=0.239, p=0.102$ ), (LC thickness $r=0.160, p=0.274$ ).

The study also looked into the prelaminar and laminar thickness of each subjects and it was found out that there was a significant difference between pre-laminar and laminar thickness $(t=12.003, p=<0.00001)$. It can be said that the prelaminar area is very dynamic and it reacts to changes in intraocular pressure acutely (Table 4). In the glaucoma group, there was no statistically significant difference in the lamina cribrosa thickness between different glaucoma severities $(f=0.406$, $p=0.672$ ). There was also no significant difference in the comparison between mild glaucoma and non-glaucomatous eyes. However, there is a statistically significant difference in the lamina cribrosa thickness between moderate glaucoma and non-glaucomatous eyes $(t=-1.974$, $p=<0.02852$ ).

\section{Discussion and Conclusion}

This study was performed to evaluate the relationship of lamina cribrosa thickness using EDI-OCT and the visual field function using Humphrey SAP in glaucomatous and non- glaucomatous eyes. The results reveal that lamina cribrosa thickness decreases as glaucoma progresses, specifically evident in subjects with moderate glaucomatous visual field loss. The researcher excluded eyes with poor visibility of 
Table 1: Modified Hodapp-Anderson-Parish criteria.

\begin{tabular}{|c|c|}
\hline Mild glaucoma & Mean deviation (MD) no worse than $6 \mathrm{~dB}$ \\
\hline & $\begin{array}{l}\text { Less than } 25 \% \text { of points depressed below the } 5 \% \\
\text { level and } 15 \% \text { of points depressed below the } 1 \% \\
\text { level }\end{array}$ \\
\hline & No point within $5^{\circ}$ with sensitivity $15 \mathrm{~dB}$ \\
\hline \multirow[t]{4}{*}{$\begin{array}{l}\text { Moderate } \\
\text { glaucoma }\end{array}$} & MD worse than $6 \mathrm{~dB}$ but no worse than $12 \mathrm{db}$ \\
\hline & $\begin{array}{l}\text { Less than } 50 \% \text { of points depressed below the } 5 \% \\
\text { level and } 25 \% \text { of points depressed below the } 1 \% \\
\text { level }\end{array}$ \\
\hline & No point within central $5^{\circ}$ with sensitivity $0 \mathrm{~dB}$ \\
\hline & $\begin{array}{l}\text { only } 1 \text { hemifield containing a point with sensitivity } \\
15 \mathrm{~dB} \text { within } 5^{\circ} \text { of fixation }\end{array}$ \\
\hline \multirow[t]{4}{*}{ Severe glaucoma } & MD worse than $12 \mathrm{~dB}$ \\
\hline & $\begin{array}{l}\text { More than } 50 \% \text { of point depressed below the } 5 \% \\
\text { level or } 25 \% \text { of points depressed below the } 1 \% \\
\text { level }\end{array}$ \\
\hline & $\begin{array}{l}\text { presence of any point within central } 5^{\circ} \text { with } \\
\text { sensitivity } 0 \mathrm{~dB}\end{array}$ \\
\hline & $\begin{array}{l}\text { Both hemifields containing point(S) with sensitivity } \\
15 \mathrm{~dB} \text { within } 5^{\circ} \text { of fixation }\end{array}$ \\
\hline
\end{tabular}

Table 2: Demographic characteristics of Subjects.

\begin{tabular}{|l|c|c|}
\multicolumn{1}{|c|}{ Age, years } & Glaucoma (22) & Non-glaucoma (26) \\
\hline Mean & 61.5 & 58.42 \\
\hline Range & $46-78$ & $46-76$ \\
\hline Sex, $\mathbf{n}$ & & \\
\hline Male (15) & 6 & 9 \\
\hline Female (13) & 5 & 8 \\
\hline
\end{tabular}

Table 3: Severity of Glaucomatous eyes.

\begin{tabular}{|l|c|}
\multicolumn{1}{|c|}{ Severity, $n$} & Subjects \\
\hline Normal & 26 \\
\hline Mild & 11 \\
\hline Moderate & 8 \\
\hline Severe & 3 \\
\hline
\end{tabular}

Table 4: Lamina Cribrosa Thickness across Different Groups.

\begin{tabular}{|l|c|c|c|c|}
\hline Lamina Cribrosa Thickness & Normal & Mild & Moderate & Severe \\
\hline Mean, um & 176.42 & 160.46 & 148.75 & 169.33 \\
\hline Std. dev. & 35.17 & 35.97 & 32.83 & 54.37 \\
\hline
\end{tabular}

lamina cribrosa, which was found in deeply excavated optic discs usually in eyes with severe glaucoma, thus also having few subjects in this glaucoma sub-group. RGC axons in the lamina cribrosa have both functional and structural support afforded by laminar beams. Blood supply passes through capillaries located in the said laminar beams [7]. Support is lost when laminar tissue is damaged, especially because they have no collateral blood supply, thus there is more evident visual field loss in patients with moderate glaucoma as these laminar beams have been further damaged or exposed to stress and became more predisposed to collapse. It was also theorized that mechanical stress to the lamina cribrosa could lead to activation of lamina cribrosa astrocytes [8] that cascades into cytokine production, antigen presentation and eventually optic neuropathy. Still further studies are needed to shed light to these concepts. Several studies involving glaucoma and nonglaucoma subjects have shown significant differences in the lamina cribrosa measurements and also different glaucoma severities will have significant differences in the measurements $[9,10]$. These correlations will be valuable as early detection and accurate assessment in the progression of glaucoma is still the ultimate goal of the different studies in this particular area. Establishment of the lamina cribrosa as a vital part of glaucoma diagnosis and management will definitely be of great value to ophthalmologists and also to engineers who are continually developing better technologies and software to help quantify clinical findings. For this reason, quantitative measurements of glaucomatous structural changes can be a good modality such as EDI-OCT of the ONH. Measurement of the lamina cribrosa characteristics with the side of the EDI- OCT afforded reproducible and accurate estimates. EDI-OCT had better agreement with SAP results. In this study, we evaluated the $\mathrm{ONH}$ through measurements in lamina cribrosa and correlating it with SAP results.

Previous time-domian (TD) OCT measurements of the $\mathrm{ONH}$ used calculations based only on interpolations of 6 radial B-scans [11]. Thus, these studies had poor reproducibility. The Cirrus EDI OCT acquires lamina cribrosa characteristics through HD-OCT Technology, which is a low-coherence interferometry that produces high-resolution tomograms from a $840 \mathrm{~nm}$ super luminescent light emitting diode [11].

This study is partially limited by the age distribution of the subjects in both glaucoma and non-glaucoma groups. The age range of glaucoma group (46-78 years) is comparable to the non-glaucoma group (48-78 years). To further correlate that lamina cribrosa changes can be age-related, a wider age range of subjects is needed. Although there have been some investigations on age-related changes of the lamina cribrosa stating that there is an increase in collagen type 112 in the laminar plates as one ages and subsequent lamina cribrosa stiffness ensues, making it more resilient to deformation. Still, more investigations are needed to clearly show the age-related effects on the lamina cribrosa.

In summary, lamina cribrosa thickness was significantly different between glaucoma and non-glaucoma groups. Upon looking into the different glaucoma severities, lamina cribrosa thickness between moderate glaucoma and non-glaucoma subjects have a statistically significant difference, it can be used as another index in fortifying glaucoma as a diagnosis. Changes in the lamina cribrosa may have not been evident in early or mild glaucoma owing to its age-related stiffness. In this study, the mean age of glaucoma subjects is at 61.50 years and 58.20 years in non-glaucoma subjects, this may have affected results having no significant difference in lamina cribrosa and prelaminar volume between non-glaucoma and mild glaucoma groups. The insignificant difference in the lamina cribrosa thickness and prelaminar volume between the non-glaucoma and severe glaucoma groups can be attributed to the small number of severe glaucoma eyes (3) included in the study.

The observer measured the lamina cribrosa characteristics prior to viewing any other data. Although masked as to the clinical information regarding the severity of glaucoma, the observer may have clues as to the subjects' disease status upon seeing the shape, configuration and thickness of the prelaminar tissue. This may have some effect on 
observer's sensitivity to the OCT images during measurement, thus findings need better equipment in viewing deeper structures such as the lamina cribrosa when technology becomes available and if possible histologic studies in the future.

This study was also limited due to a small sample size especially in patients with severe glaucomatous visual field loss, short period of study, and relatively beginning learning curve of the diagnostic support staff in capturing the lamina cribrosa using the current EDIOCT equipment and software. Recommendations would include making ONH OCT routine, longer study duration with serial followups, multi-center study, age-matched subjects, use of multiple SAP with full threshold 24-2 SITA strategy and acquiring images using a superior equipment such as the Spectralis OCT (Heidelberg Engineering, GmbH, Dossenheim, Germany) that captures a [1015] degree rectangle for horizontal scans centered on the optic disc, which scans 97 sections, and each section had 20 OCT frames averaged. Progressive longitudinal studies are needed to investigate the relationship between lamina cribrosa characteristics and visual field loss progression.

\section{References}

1. Kingman S(2004) Glaucoma is second leading cause of blindness globally. Bull World Health Organ 82: 811-890.

2. Cubillan LDP, Olivar-Santos EO (2005) Third national survey on blindness. Philipp J Ophthal 30: 100-114.

3. Kaw SMG, Martinez JM, Tumbocon JA, Atienza NJ (2012) Correlation of average RNFL thickness using the STRATUS OCT with the perimetric staging of glaucoma. Philipp J Ophthalmol 37: 19-23.

4. Schlamp C L, Li Y, Dietz J A, Janssen K T, Nickells R W (2006) Progressive ganglion cell loss and optic nerve degeneration in DBA/2J mice is variable and asymmetric. BMC Neurosci 7: 66.

5. Rebolleda G, Munoz Negrete F J (2014) Enhanced Depth Imagingoptical coherence tomography technique and the lamina cribrosa in glaucoma. arch soc esp oftalmol 89: 133-135.
6. Brown DJ, Morishige N, Neekhra A, Minckler DS, Jester JV (2007) Application of second harmonic imaging microscopy to assess structural changes in optic nerve head structure ex vivo. J Biomed Opt 12: 024029.

7. Inoue R, Hangai $M$, Kotera $Y$, Nakanishi $H$, Mori $S$ et al. (2009) Three-dimensional high-speed optical coherence tomography imaging of lamina cribrosa in glaucoma. Ophthalmology 116: 214222.

8. Kotecha A, Izadi S, Jeffery G (2006) Age-related changes in the thickness of the human lamina cribrosa. Br J Ophthalmol 90: 15311534.

9. Albon J, Karwatowski WS, Easty DL, Sims TJ, Duance VC (2000) Age related changes in the non-collagenous components of the extracellular matrix of the human lamina cribrosa. Br J Ophthalmol 84: 311-317.

10. Morrison JC, Jerdan JA, Dorman ME, Quigley HA (1989) Structural proteins of the neonatal and adult lamina cribrosa. Arch Ophthalmol 107: $1220-1224$.

11. Hernandez MR, Luo XX, Andrzejewska W, Neufeld AH (1989) Agerelated changes in the extracellular matrix of the human optic nerve head. Am J Ophthalmol 107: 476-484.

12. Park HY, Jeon SH, Park CK (2012) Enhanced depth imaging detects lamina cribrosa thickness differences in normal tension glaucoma and primary open-angle glaucoma. Ophthalmology 119: 10-20.

13. Agoumi Y, Sharpe GP, Hutchison DM, Nicolela MT, Artes PH, et al. (2011) Laminar and prelaminar tissue displacement during intraocular pressure elevation in glaucoma patients and healthy controls. Ophthalmology 118: 52-59.

14. Lee EJ, Kim TW, Weinreb RN, Kim H (2013) Reversal of lamina cribrosa displacement after intraocular pressure reduction in openangle glaucoma. Ophthalmology 120: 553-559.

15. Park SC, de Moraes CG, Teng CC, Tello C, Liebmann JM, et al. (2012) Enhanced depth imaging optical coherence tomography of deep optic nerve complex structures in glaucoma. Ophthalmology 119: 3-9. 